\title{
Cardiac PET/CT Misregistration Causes Significant Changes in Estimated Myocardial Blood Flow
}

\author{
Mahadevan Rajaram ${ }^{1}$, Abdel K. Tahari ${ }^{1}$, Andy H. Lee ${ }^{1}$, Martin A. Lodge ${ }^{1}$, Benjamin Tsui ${ }^{1}$, Stephan Nekolla ${ }^{2}$, \\ Richard L. Wahl ${ }^{1}$, Frank M. Bengel ${ }^{3}$, and Paco E. Bravo ${ }^{1}$ \\ ${ }^{1}$ Department of Radiology, Johns Hopkins Medical Institutions, Baltimore, Maryland; ${ }^{2}$ Technical University of Munich, \\ Munich, Germany; and ${ }^{3}$ Department of Nuclear Medicine, Hannover Medical School, Hannover, Germany
}

\begin{abstract}
Misregistration of cardiac PET/CT data can lead to misinterpretation of regional myocardial perfusion. However, the effect of misregistration on the quantification of myocardial blood flow (MBF) has not been studied. Methods: Cardiac ${ }^{82} \mathrm{Rb}-\mathrm{PET} / \mathrm{CT}$ scans of 10 patients with normal regional myocardial perfusion were analyzed. Realignment was done for the baseline and stress PET/CT images as necessary, and MBF was obtained from dynamic data. Then, the stress images were misregistered by $5 \mathrm{~mm}$ along the $x$-axis (left) and $z$-axis (cranial) and again by $10 \mathrm{~mm}$. A 10-mm misregistration in the opposite direction $(-10 \mathrm{~mm}$ along the $x$-axis [right] and $z$-axis [caudal]) was also tested. Stress MBF was recalculated for 5-, 10-, and -10-mm misregistrations. Results: Stress MBF of the left ventricle decreased by $10 \% \pm 6 \%(P=0.005)$ after $5-\mathrm{mm}$ misregistration and by $24 \% \pm 15 \%(P=0.001)$ after $10-\mathrm{mm}$ misregistration. In descending order, the most important stress MBF changes occurred in the anterior $(39 \% \pm 9 \%)$, lateral $(34 \% \pm 9 \%)$, apical $(20 \% \pm 16 \%)$, inferior $(12 \% \pm 10 \%)$, and septal $(10 \% \pm 12 \%)$ walls after $10-\mathrm{mm}$ misregistration. Lesser changes were observed after 5-mm misregistration, with the same wall distribution. In contrast, $-10-\mathrm{mm}$ misregistration increased global MBF by $9 \% \pm 6 \%(P=0.004)$. In descending order, the overestimation of estimated MBF after - 10-mm misregistration occurred in the lateral $(15 \% \pm 8 \%)$, apical $(15 \% \pm 18 \%)$, anterior $(9 \% \pm 5 \%)$, and inferior $(9 \% \pm 11 \%)$ walls. Conclusion: Misregistration of the stress PET/CT dataset leads to significant global and regional artifactual alterations in the estimated MBF. Quantitative error was observed throughout the myocardium and was not confined to those heart regions that extended into the lung on misregistered CT.
\end{abstract}

Key Words: PET/CT misregistration; absolute myocardial blood flow; myocardial flow reserve

J Nucl Med 2013; 54:50-54

DOI: 10.2967/jnumed.112.108183

Received May 2, 2012; revision accepted Jul. 20, 2012.

For correspondence or reprints contact: Paco E. Bravo, Division of Nuclear Medicine, Johns Hopkins University, 601 N. Caroline St., Ste. 3225, Baltimore, MD 21287.

E-mail: pbravov1@jhmi.edu

Published online Oct. 22, 2012.

COPYRIGHT (C) 2013 by the Society of Nuclear Medicine and Molecular Imaging, Inc. ments uantitative cardiovascular PET, including measurements of absolute myocardial blood flow (MBF) and myocardial flow reserve (MFR), seems likely to become part of routine clinical practice in the evaluation of individuals with heart disease. There is supportive evidence demonstrating that impaired vasodilator-induced MBF and MFR are independent risk factors for cardiac death and other adverse cardiovascular events (1-4). Precise and reliable quantification of flow is of paramount importance in the management of these patients.

It has previously been demonstrated that accurate estimation of myocardial tracer uptake for regional myocardial perfusion PET requires proper alignment of the emissiontransmission data (5). Error-free reconstruction of PET data with a registered $\mathrm{CT}$ attenuation map is essential for accurate quantification and interpretation of cardiac perfusion.

Misalignment of the CT and PET studies can be caused by respiratory or cardiac motion and gross physical movement of the patient. This motion can produce an erroneous attenuation map that imposes lung attenuation parameters onto the heart wall, thereby underestimating the attenuation and creating artifactual areas of hypoperfusion $(5,6)$. Moreover, regional inhomogeneities in attenuation produced by misregistration are frequently not appreciated visually but may be important quantitatively and need to be considered. The impact of emission-transmission misregistration of cardiac PET/CT datasets on MBF quantification has not, to our knowledge, been evaluated yet and was the aim of the present study.

\section{MATERIALS AND METHODS}

\section{Study Group}

Cardiac PET/CT image datasets of 10 patients referred for clinical indications were analyzed. To avoid interfering effects of flow-limiting coronary stenosis, only subjects with no prior history of coronary artery disease who had normal regional myocardial perfusion and an MFR greater than 3.00 on PET were included in this analysis. The study was approved by the ethics committee, and the need to obtain informed consent was waived.

\section{PET/CT Protocol}

All imaging was performed on a 64-slice Discovery Rx VCT PET/ CT scanner (GE Healthcare). Individuals were positioned, and a lowdose helical CT scan $(120 \mathrm{kV}, 50-100 \mathrm{~mA})$ was acquired during shallow breathing before rest imaging for attenuation correction 
of the rest and stress PET data. Through a large antecubital intravenous line, $1,480-1,850 \mathrm{MBq}$ of ${ }^{82} \mathrm{Rb}$ were infused, and a listmode 2-dimensional PET scan was acquired for $8 \mathrm{~min}$.

Vasodilator stress with dipyridamole $(0.56 \mathrm{mg} / \mathrm{kg}, 4 \mathrm{~min})$ was started after the rest acquisition, and a second dose of 1,480-1,850 $\mathrm{MBq}$ of ${ }^{82} \mathrm{Rb}$ was injected, starting 4 min after the end of the dipyridamole infusion and followed by acquisition of an 8-min list-mode scan. The patient was not moved between the rest and stress scans.

\section{PET/CT Misregistration}

Manual registration was created by interactively moving the CT image over the PET image in the coronal, sagittal, and transaxial views. Four sets of cardiac PET/CT images were obtained. The first set was assigned to the no-misregistration group. Both rest and stress PET/CT images were visually aligned for proper registration, paying special attention to ensure that the left ventricular myocardial activity on PET did not overlap with the lung parenchyma on CT. The second set included only stress images in which a 5-mm misregistration of the PET and CT datasets was artificially created along both the $x$-axis (left) and the $z$-axis (cranial), such that there was overlap between the lung parenchyma on CT and the lateral and anterior walls of the left ventricle on PET. The third set included only stress images with $10-\mathrm{mm}$ misregistration along both the $x$ - and the $z$-axes, resulting in a similar overlap pattern between the lung and the heart. The fourth set included images in which a $10-\mathrm{mm}$ misregistration was artificially created in the opposite direction ( $-10-\mathrm{mm}$ misregistration), that is, $10 \mathrm{~mm}$ toward the right along the $x$-axis and $10 \mathrm{~mm}$ caudally along the z-axis (Fig. 1).

Separate attenuation-corrected PET images based on these 4 different registration conditions were generated. For each registration condition, static (90-s prescan delay) and dynamic (32 frames: $20 \times 6 \mathrm{~s}, 5 \times 12 \mathrm{~s}, 4 \times 30 \mathrm{~s}$, and $3 \times 60 \mathrm{~s}$ ) images were reconstructed using the ordered-subsets expectation-maximization algorithm (2 iterations, 21 subsets).

\section{Image Analysis}

Quantification was performed using the custom-designed MunichHeart software, which had been previously validated at our institution (7). Myocardial activity in the last frame of the dynamic datasets was volumetrically sampled, and segmented polar maps of the left ventricle were generated and applied to the

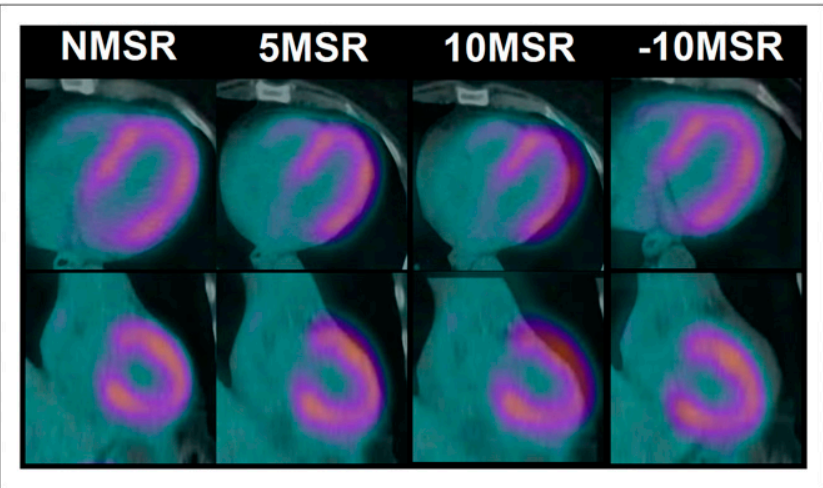

FIGURE 1. Axial (top row) and coronal (bottom row) images showing no misregistration (NMSR) and effects of 5-mm misregistration (5MSR), 10-mm misregistration (10MSR), and reverse 10-mm misregistration $(-10 \mathrm{MSR})$ between $\mathrm{PET}$ and $\mathrm{CT}$ images. whole dynamic series to obtain myocardial time-activity curves. A small region of interest was positioned in the left ventricular cavity to obtain the arterial input function. MBF was then quantified using a simplified retention approach $(7,8)$. The concentration of myocardial activity between 4 and $8 \mathrm{~min}$ was normalized to the area under the arterial input function in the first $120 \mathrm{~s}$. The resulting index was software-corrected for partial volume, spillover, and nonlinear extraction of ${ }^{82} \mathrm{Rb}(7)$.

This process was initially performed on the no-misregistration dataset and then repeated on the 5- and 10-mm misregistration datasets.

MBF was obtained at peak stress and rest, and the ratio of stress flow to rest flow was used to obtain MFR. Both MBF and MFR were obtained on a global and regional basis for different walls of the left ventricle (anterior, inferior, lateral, septal, and apical) in all 3 sets of images. Additionally, the summed stress score was calculated using the standard 17-segment 5-point scale (9).

\section{Statistical Analysis}

Data are shown as mean \pm SD or mean percentage change and 95\% confidence interval (CI). An ANOVA with repeated measures combined with the Bonferroni test for post hoc analysis and correction for multiple comparisons was used to compare global and regional differences in flow between no misregistration and 5- and 10 -mm misregistration. $P$ values of less than 0.05 were considered statistically significant. Analysis was performed using SPSS (version 19.0; IBM) for Windows (Microsoft).

\section{RESULTS}

Regional myocardial perfusion was normal at baseline (summed stress score, 0 in all no-misregistration cases) but changed significantly with 5 -mm misregistration (summed stress score, $2.4 \pm 1.2 ; P<0.0001$ ) and 10 -mm misregistration (summed stress score, $8.0 \pm 2.3 ; P<0.0001$ ) after development of apparent regional defects in the anterior and lateral walls (Fig. 2).

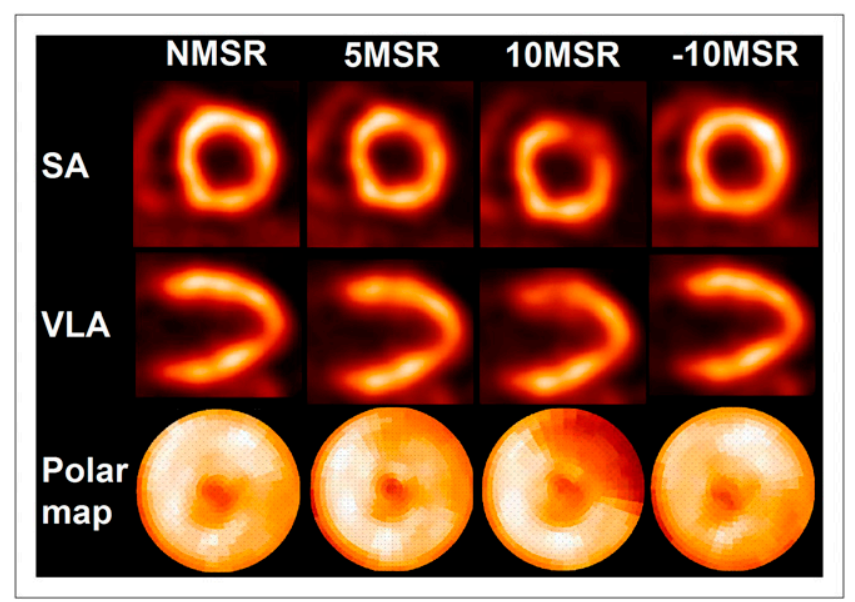

FIGURE 2. Myocardial perfusion stress PET images and polar maps demonstrate effects of no misregistration (NMSR) and effects of 5-mm misregistration (5MSR), 10-mm misregistration (10MSR), and reverse $10-\mathrm{mm}$ misregistration (-10MSR) between PET and CT images. Artifactual defects are prominent in anterior and lateral walls of heart, although this patient had no disease. $S A=$ short axis; VLA $=$ vertical long axis. 
TABLE 1

Regional and Global Differences in Calculation of Vasodilator-Induced Stress MBF

\begin{tabular}{|c|c|c|c|c|c|c|c|}
\hline \multirow[b]{2}{*}{ Region } & \multicolumn{4}{|c|}{ Misregistration $(\mathrm{mL} / \mathrm{min} / \mathrm{g})$} & \multicolumn{3}{|c|}{$P$ vs. misregistration } \\
\hline & None & $5 \mathrm{~mm}$ & $10 \mathrm{~mm}$ & $-10 \mathrm{~mm}$ & $5 \mathrm{~mm}$ & $10 \mathrm{~mm}$ & $-10 \mathrm{~mm}$ \\
\hline Anterior & $2.82 \pm 0.54$ & $2.36 \pm 0.45$ & $1.75 \pm 0.5$ & $3.06 \pm 0.56$ & $<0.0001$ & $<0.0001$ & 0.001 \\
\hline Septal & $2.47 \pm 0.51$ & $2.41 \pm 0.45$ & $2.22 \pm 0.59$ & $2.40 \pm 0.50$ & 0.9 & 0.2 & 0.9 \\
\hline Inferior & $2.33 \pm 0.57$ & $2.21 \pm 0.56$ & $2.05 \pm 0.61$ & $2.52 \pm 0.54$ & 0.1 & 0.04 & 0.1 \\
\hline Lateral & $2.73 \pm 0.55$ & $2.35 \pm 0.42$ & $1.80 \pm 0.44$ & $3.12 \pm 0.56$ & 0.002 & $<0.0001$ & 0.001 \\
\hline Apical & $2.01 \pm 0.41$ & $1.84 \pm 0.44$ & $1.61 \pm 0.47$ & $2.29 \pm 0.39$ & 0.2 & 0.04 & 0.01 \\
\hline Total left ventricle & $2.47 \pm 0.46$ & $2.23 \pm 0.41$ & $1.88 \pm 0.47$ & $2.67 \pm 0.45$ & 0.005 & 0.001 & 0.004 \\
\hline
\end{tabular}

Between the no-misregistration and 10-mm misregistration studies, there was a statistically significant decrease in stress-induced global MBF (24\% change; 95\% CI, 17-31; $P=0.001)$ and global MFR (23\% change; $95 \%$ CI, 15-30; $P<0.0001)$. The reduction in stress-induced global MBF (10\% change; $95 \%$ CI, 5-14; $P=0.005$ ) and global MFR (9\% change; $95 \% \mathrm{CI}, 5-13 ; P=0.02$ ) between the nomisregistration and 5-mm misregistration datasets was also significant. There was an overestimation of global MBF (9\% change; $95 \%$ CI, 3-13; $P=0.004)$ and MFR $(12 \%$ change; $95 \% \mathrm{CI}, 2-21 ; P=0.2)$ after -10 -mm misregistration (Tables 1 and 2).

On a regional basis, and in descending order of change, stress MBF (Fig. 3) and MFR decreased in the anterior, lateral, apical, inferior, and septal walls after both 5- and 10-mm misregistration compared with no misregistration. This reduction was statistically significant in all myocardial walls (except the septum for stress MBF) between no-misregistration and 10-mm misregistration datasets but reached statistical significance only for the anterior and lateral walls between the no-misregistration and 5-mm misregistration studies (Table 1). In the $-10-\mathrm{mm}$ misregistration images, regional MBF was significantly overestimated (in descending order of change) in the lateral, apical, and anterior walls (Tables 1 and 2; Fig. 3).

\section{DISCUSSION}

The main finding of our study was that misregistration of the stress PET/CT dataset caused a marked global and regional reduction of the calculated absolute flow. Although this was somewhat expected, the magnitude of the differ- ence in stress MBF and MFR due to varying degrees of PET/ CT misregistration had not been previously determined.

Misregistration of CT for attenuation correction and PET emission data with associated artifactual PET defects is due to momentary helical CT snapshots at some point in the respiratory cycle. The attenuation of thoracicdiaphragmatic structures on the snapshot CT scan may not match the actual average attenuation of the constantly changing thoracic-diaphragmatic structures over longer emission scans during normal breathing $(5,10)$. A study by Gould et al. showed that most misregistration defects were anterior or lateral and were associated with corresponding anterior or lateral misregistration on the PET/CT fusion images (5). The marked difference in the attenuation factor of cardiac and lung tissue has been mentioned as the possible reason for the changes in measured tracer uptake (10).

To compute absolute flow, several corrections have to be made to the data, including correcting the myocardial counts for partial-volume losses and for spillover from blood-pool activity to the myocardium, and correction of blood-pool activity or arterial input for myocardial-toblood-pool spillover (8). All these factors may potentially be affected by artifactual myocardial perfusion defects caused by misregistration, leading to false absolute MBF values. Therefore, measurements of absolute MBF may be appropriate only if the primary radionuclide uptake data are correctly obtained using properly registered PET/CT images. Similar corrections are required when MBF is calculated using ${ }^{13} \mathrm{~N}$-ammonia $(11,12)$, and it is likely that misregistration between the emission and transmission images

TABLE 2

Regional and Global Differences in Calculation of MFR

\begin{tabular}{|c|c|c|c|c|c|c|c|}
\hline \multirow[b]{2}{*}{ Region } & \multicolumn{4}{|c|}{ Misregistration $(\mathrm{mL} / \mathrm{min} / \mathrm{g})$} & \multicolumn{3}{|c|}{$P$ vs. misregistration } \\
\hline & None & $5 \mathrm{~mm}$ & $10 \mathrm{~mm}$ & $-10 \mathrm{~mm}$ & $5 \mathrm{~mm}$ & $10 \mathrm{~mm}$ & $-10 \mathrm{~mm}$ \\
\hline Anterior & $4.32 \pm 0.77$ & $3.63 \pm 0.51$ & $2.70 \pm 0.70$ & $4.70 \pm 0.86$ & $<0.0001$ & $<0.0001$ & 0.004 \\
\hline Septal & $4.04 \pm 0.67$ & $3.92 \pm 0.54$ & $3.62 \pm 0.85$ & $3.89 \pm 0.48$ & 0.9 & 0.07 & 0.9 \\
\hline Inferior & $3.80 \pm 0.54$ & $3.59 \pm 0.56$ & $3.37 \pm 0.73$ & $4.14 \pm 0.76$ & 0.2 & 0.02 & 0.3 \\
\hline Lateral & $3.79 \pm 0.59$ & $3.25 \pm 0.49$ & $2.51 \pm 0.47$ & $4.34 \pm 0.70$ & 0.004 & $<0.0001$ & 0.003 \\
\hline Apical & $4.07 \pm 0.60$ & $3.71 \pm 0.69$ & $3.40 \pm 1.01$ & $4.66 \pm 0.70$ & 0.2 & 0.07 & 0.02 \\
\hline Total left ventricle & $3.99 \pm 0.55$ & $3.62 \pm 0.46$ & $3.11 \pm 0.65$ & $4.49 \pm 1.03$ & 0.02 & $<0.0001$ & 0.2 \\
\hline
\end{tabular}




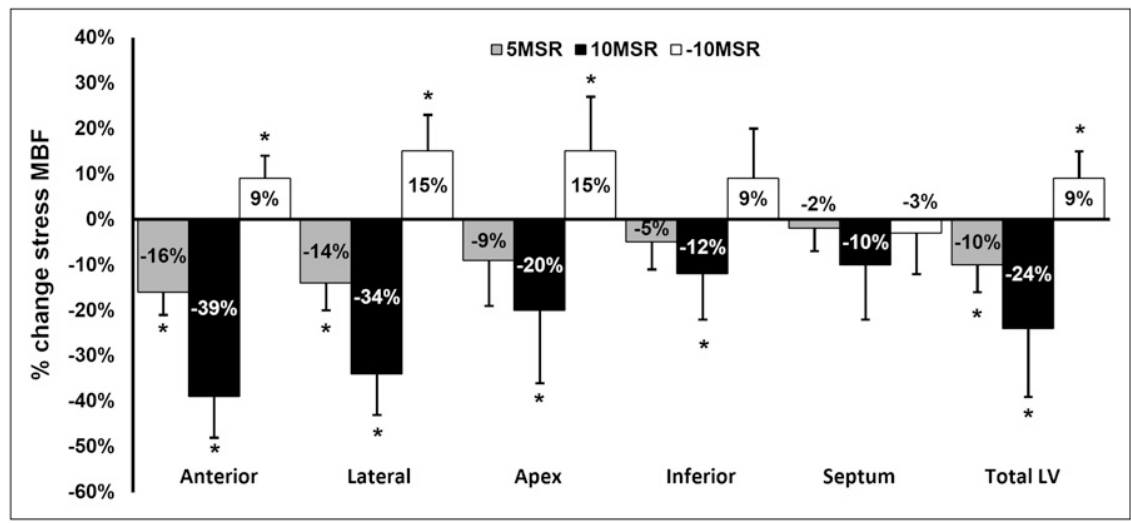

FIGURE 3. Percentage decrease in artifactual stress MBF (\% change \pm SD) to different ventricular walls and total left ventricle with 5-mm misregistration (5MSR), 10-mm misregistration (10MSR), and reverse 10-mm misregistration (-10MSR) datasets compared with images with no misregistration. *Statistically significant changes in estimates of absolute stress MBF compared with images with no misregistration. will affect MBF estimation when ${ }^{13} \mathrm{~N}$-ammonia is used as a radiotracer.

In this study, to avoid unnecessary radiation exposure, we obtained a single CT scan for attenuation correction (before rest imaging) of both rest and stress PET datasets since we previously found that a repeated CT scan after the stress study does not significantly improve misregistration in the stress images (6).

There has been renewed interest in translation of $\mathrm{MBF}$ and MFR using PET from the research realm to routine clinical practice. Recent studies have shown significant diagnostic potential for abnormal MFR in identification of triple-vessel disease and the prognostic impact of abnormal MFR over and above that predicted by traditional risk factors and high-risk findings on a cardiac PET/CT scan (1-4). With the increasing availability of PET, there is a growing potential for wider application of this measurement. Our study shows that even a $0.5-\mathrm{cm}$ misregistration of the anterior and lateral walls can decrease estimates of stress-induced global MBF by $5 \%-14 \%$ and that $1 \mathrm{~cm}$ of misregistration can decrease it by a factor of $17 \%-31 \%$. Importantly, misregistration affected the measured regional flow in all myocardial walls, including the inferior wall and septum, despite no obvious overlap between these walls on PET and tissues of different attenuation densities on CT. This quantitative error was observed throughout the myocardium because of the way photon attenuation affects PET images. The attenuation at a particular point is dependent on the total attenuation along all lines of response passing through that point, not simply on the attenuation properties at that immediate location. As such, attenuation correction factors derived from misregistered CT will be expected to be inaccurate for all locations, not simply those areas where there is a pronounced difference in tissue attenuation, such as when myocardium on PET is seen to lie in the lung on CT. This was seen in our study, with the greatest differences occurring in the anterior and lateral walls, along with a lesser degree of calculated differences in the inferior wall and septum. These differences became more pronounced with increasing severity of misregistration. Interestingly, when the PET images in our study were misregistered by
$10 \mathrm{~mm}$ in the opposite direction, there was a statistically significant overestimation of MBF. However, the estimated change was to a lesser degree than that seen after $10-\mathrm{mm}$ misregistration, most likely because of increased attenuation correction factors applied to the central region of the field of view. This possibility is supported by the fact that the change was least in the septum (located more centrally in the field of view) and maximal at the apex and the lateral wall, which are more peripherally located.

The limitations of this study were that only a small number of subjects without a history of coronary artery disease were studied, and only a single validated model of MBF estimation was used. Perfusion using ${ }^{82} \mathrm{Rb}$ has been validated for estimation of MBF using different analytic methods. The magnitude of change that could potentially occur during misregistration using other validated models for estimation of $\mathrm{MBF}$ with ${ }^{82} \mathrm{Rb}$ has not yet been studied.

\section{CONCLUSION}

Misregistration in the axial and coronal planes of stress PET/CT studies causes significant artifactual errors in estimation of global and regional myocardial flow. Quantitative error was observed throughout the myocardium and was not confined to those heart regions that extended into the lung on misregistered CT. This study reinforces the need for appropriate quality control and for care to ensure optimal registration when cardiac PET/CT images are obtained for either clinical or investigational purposes.

\section{DISCLOSURE}

The costs of publication of this article were defrayed in part by the payment of page charges. Therefore, and solely to indicate this fact, this article is hereby marked "advertisement" in accordance with 18 USC section 1734. No potential conflict of interest relevant to this article was reported.

\section{ACKNOWLEDGMENTS}

We thank Judy Buchanan from the Division of Nuclear Medicine at Johns Hopkins for her helpful editorial assistance. 


\section{REFERENCES}

1. Ziadi MC, Dekemp RA, Williams KA, et al. Impaired myocardial flow reserve on rubidium-82 positron emission tomography imaging predicts adverse outcomes in patients assessed for myocardial ischemia. J Am Coll Cardiol. 2011; 58:740-748

2. Fukushima K, Javadi MS, Higuchi T, et al. Prediction of short-term cardiovascular events using quantification of global myocardial flow reserve in patients referred for clinical ${ }^{82} \mathrm{Rb}$ PET perfusion imaging. J Nucl Med. 2011; 52:726-732.

3. Murthy VL, Naya M, Foster CR, et al. Improved cardiac risk assessment with noninvasive measures of coronary flow reserve. Circulation. 2011;124:22152224.

4. Ziadi MC, Dekemp RA, Williams K, et al. Does quantification of myocardial flow reserve using rubidium- 82 positron emission tomography facilitate detection of multivessel coronary artery disease? J Nucl Cardiol. 2012;19:670-680.

5. Gould KL, Pan T, Loghin C, Johnson NP, Guha A, Sdringola S. Frequent diagnostic errors in cardiac PET/CT due to misregistration of CT attenuation and emission PET images: a definitive analysis of causes, consequences, and corrections. J Nucl Med. 2007;48:1112-1121.

6. Lautamäki R, Brown TL, Merrill J, Bengel FM. CT-based attenuation correction in ${ }^{82} \mathrm{Rb}$-myocardial perfusion PET-CT: incidence of misalignment and effect on regional tracer distribution. Eur J Nucl Med Mol Imaging. 2008; 35:305-310.

7. Lautamäki R, George RT, Kitagawa K, et al. Rubidium-82 PET-CT for quantitative assessment of myocardial blood flow: validation in a canine model of coronary artery stenosis. Eur J Nucl Med Mol Imaging. 2009;36: 576-586.

8. Yoshida K, Mullani N, Gould KL. Coronary flow and flow reserve by PET simplified for clinical applications using rubidium-82 or nitrogen-13-ammonia. J Nucl Med. 1996;37:1701-1712.

9. Taillefer R, Ahlberg AW, Masood Y, et al. Acute beta-blockade reduces the extent and severity of myocardial perfusion defects with dipyridamole Tc-99m sestamibi SPECT imaging. J Am Coll Cardiol. 2003;42:1475-1483.

10. Martinez-Moller A, Souvatzoglou M, Navab N, Schwaiger M, Nekolla SG. Artifacts from misaligned CT in cardiac perfusion PET/CT studies: frequency, effects, and potential solutions. J Nucl Med. 2007;48:188-193.

11. Kuhle WG, Porenta G, Huang SC, et al. Quantification of regional myocardial blood flow using ${ }^{13} \mathrm{~N}$-ammonia and reoriented dynamic positron emission tomographic imaging. Circulation. 1992;86:1004-1017.

12. Muzik O, Beanlands RS, Hutchins GD, Mangner TJ, Nguyen N, Schwaiger M. Validation of nitrogen-13-ammonia tracer kinetic model for quantification of myocardial blood flow using PET. J Nucl Med. 1993;34: 83-91. 